\title{
WIDE-LINE MOLECULAR CLOUDS AND THE GAMMA-RAY DEFICIT TOWARD THE GALACTIC CENTER
}

\author{
J. G. STACY ${ }^{1}$, M. E. BITRAN ${ }^{2}$, T. M. DAME ${ }^{1}$, AND P. THADDEUS ${ }^{1}$ \\ ${ }^{1}$ Harvard-Smithsonian Center for Astrophysics \\ 60 Garden St., Cambridge, MA 02138 USA \\ ${ }^{2}$ Department of Astronomy \\ University of Florida, Gainesville, FL 92611 USA
}

\begin{abstract}
The discrepancy between observed and predicted $\boldsymbol{\gamma}$-ray emission toward the Galactic Center is attributed to a unique population of wide-line molecular clouds. The most prominent objects of this class show evidence of rotation and a significant stellar population. The observed ${ }^{12} \mathrm{CO}$ emission traces the gravitational field produced primarily by stars, not molecular gas.
\end{abstract}

\section{Introduction}

The correlation between diffuse, Galactic, high-energy $\boldsymbol{\gamma}$-ray emission and gas tracers of the interstellar medium (e.g., CO and HI) is well-established (Hartman et al. 1979, Strong et al. 1988). The one region of notable exception is the Galactic Center, where an order of magnitude discrepancy between observed and predicted $\gamma$-ray flux has been reported (e.g., Blitz et al. 1985). We have undertaken a reanalysis of this question using a more fully-sampled, wide-latitude CO survey of the Center region (Bitran 1987), and the COS-B $\gamma$-ray data base (Mayer-Hasselwander 1985).

\section{Wide-line Molecular Clouds}

One of the most striking features of the Galactic Center CO survey is the presence of a unique population of wide-line molecular clouds (=WLC's, $<v_{\mathrm{rms}}>\sim 20-60 \mathrm{~km} \mathrm{~s}^{-1}$, Bitran et al. 1988). We have identified the overestimate in predicted $\gamma$-ray emission with the WLC's, the eight most prominent of which contribute approximately $58 \%$ of the total integrated CO intensity, $W_{C O}$, in the vicinity of the Galactic Center (Stacy et al. 1987). In order to investigate those cloud properties which may affect the molecular mass estimates of these objects, we have selected as archetypes the $3^{\circ}$ (= "Clump $\left.2^{\prime \prime}\right)$ and $5^{\circ}$ WLC's, which are the most prominent and well-defined objects of their class.

We find evidence for rotation in both the $3^{\circ}$ and $5^{\circ}$ WLC's, based on maps of emissionweighted mean velocity (see Figure 1 ). Model fits to the data yield velocity gradients of 0.6 to $0.8 \mathrm{~km} \mathrm{~s}^{-1} \mathrm{pc}^{-1}$, with projected rotation axes inclined by $\sim 40-50^{\circ}$ with respect to the Galactic plane. These values indicate rotational line widths of $\sim 60 \mathrm{~km} \mathrm{~s}^{-1}$, implying that about half the observed line width is due to ordered, rotational motion.

We also find that the $3^{\circ}$ and $5^{\circ}$ WLC's contain a significant population of old stars, deduced from large-scale surveys of $2.4 \mu \mathrm{m}$ emission toward the Galactic Center (e.g., Melnick et al. 1987). The total NIR brightness of the $3^{\circ}$ and $5^{\circ}$ clouds is estimated to be 
at the level of $\sim 1-2 \%$ that observed for the nuclear disk itself, implying stellar masses of $\sim 10^{8} \mathrm{M}_{\odot}$ (assuming $\mathrm{M} / \mathrm{L}_{2.4 \mu \mathrm{m}} \sim 1-3 \mathrm{M}_{\odot} / \mathrm{L}_{\odot}$ ).
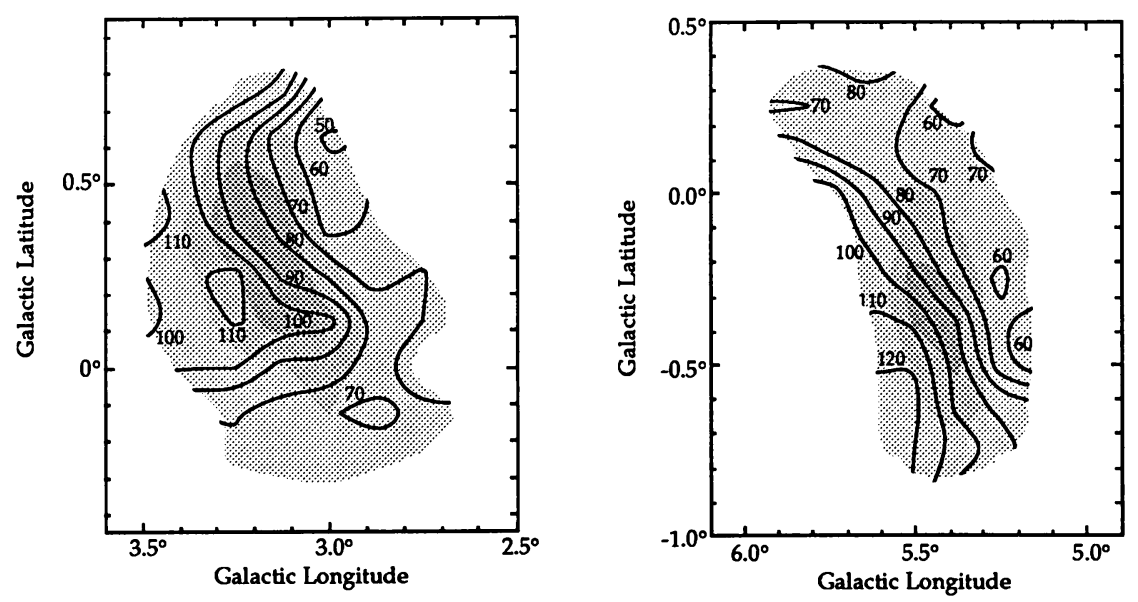

Figure 1. Emission-weighted mean velocity contours (in $\mathrm{km} \mathrm{s}^{-1}$ ) superposed on grey scale maps of integrated ${ }^{12} \mathrm{CO}$ antenna temperature for the $3^{\circ}$ (left) and $5^{\circ}$ (right) molecular clouds (grey scale levels: $175,375,575 \mathrm{~K} \mathrm{~km} \mathrm{~s}^{-1}$ and $50,150,250 \mathrm{~K} \mathrm{~km} \mathrm{~s}^{-1}$ for the $3^{\circ}$ and $5^{\circ}$ clouds, respectively).

\section{Implications for $\boldsymbol{\gamma}$-ray Emission}

We conclude that the observed ${ }^{12} \mathrm{CO}$ gas traces the gravitational field produced primarily by stars, not molecular gas. The combination of optically-thin ${ }^{12} \mathrm{CO}$ emission (implied by the large velocity gradients) and possible enhanced metallicity effects (due to evolved stars) argues for a lower mass conversion ratio, $\mathrm{N}_{\mathrm{H}_{2}} / \mathrm{W}_{\mathrm{CO}}$, for these objects, which may be sufficient to reduce the molecular mass of these clouds to the extent that the $\gamma$-ray emission per nucleon is comparable to that in the outer Galaxy, i.e., effectively removing the $\gamma$-ray deficiency. Observations with the next generation of $\gamma$-ray satellite experiments (e.g., the Gamma Ray Observatory) should be capable of confirming the wide-line cloud origin of the $\boldsymbol{\gamma}$-ray deficit.

\section{References}

Bitran, M. E. 1987, Ph.D. Thesis, University of Florida.

Bitran, M. E., Thaddeus, P., and Cohen, R. S. 1988, in preparation.

Blitz, L., Bloemen, J. B. G. M., Hermsen, W., and Bania, T. M. 1985, Astron. Astrophys., 143, 267.

Hartman, R. C. et al. 1979, Ap. J., $230,597$.

Mayer-Hasselwander, H. A. 1985, Explanatory Supplement to the COS-B Data Base.

Melnick, G. J. et al. 1987, in The Galactic Center, ed. D. C. Backer (New York: AIP), 157.

Stacy, J. G., Dame, T. M., and Thaddeus, P. 1987, Proc. 20th International Cosmic Ray Conf. (Moscow), 1, 117.

Strong, A. W. et al. 1988, Astron. Astrophys., in press. 